\title{
Satisfacción laboral y compromiso organizacional en docentes de la Amazonía peruana
}

\author{
Edwin Gustavo Estrada Araozi \\ Universidad Nacional Amazónica de Madre de Dios, Puerto Maldonado, Tambopata, Perú \\ Néstor Antonio Gallegos Ramosii(i) \\ Universidad Nacional Amazónica de Madre de Dios, Puerto Maldonado, Tambopata, Perú
}

\begin{abstract}
Resumen
La presente investigación buscó establecer la relación entre la satisfacción laboral y el compromiso organizacional en los docentes de educación básica regular de la Amazonía peruana. La investigación tuvo un enfoque cuantitativo y su diseño fue no experimental de tipo correlacional. La muestra estuvo conformada por 185 docentes, a quienes se les aplicó la Escala de Satisfacción Laboral y el Cuestionario de Compromiso Organizacional, instrumentos con la validez y confiabilidad requeridas. Los hallazgos indican que el nivel de satisfacción laboral y compromiso organizacional de los docentes es regular y se determinó la existencia de una relación directa y significativa entre la satisfacción laboral y el compromiso organizacional ( $r s=0,539 ; p=0,000<0,05)$. Se concluye que, cuanto más satisfechos estén los docentes en sus centros de labores, su nivel de compromiso hacia la institución será mayor.
\end{abstract}

\section{Palabras clave}

Satisfacción del docente. Compromiso social del docente. Condiciones de trabajo docente. Docentes. Educación básica.

\section{Satisfação no trabalho e compromisso organizacional em professores da Amazônia peruana}

\begin{abstract}
Resumo
A presente pesquisa buscou estabelecer a relação entre satisfação no trabalho e comprometimento organizacional em professores regulares da educação básica na Amazônia peruana. A pesquisa teve abordagem quantitativa e seu delineamento foi não experimental do tipo correlacional. A amostra foi composta por 185 professores, aos quais foram aplicados a Escala de Satisfação no Trabalho e o Questionário de Compromisso Organizacional, instrumentos com a validade e confiabilidade exigidas. Os resultados indicam que o nível de satisfação no trabalho e comprometimento organizacional dos professores é regular e foi determinada a existência de uma relação direta e significativa entre satisfação no trabalho e comprometimento organizacional ( $r s=0,539 ; p=0,000$ $<0,05)$. Conclui-se que, quanto mais satisfeitos os docentes estão em seus centros de trabalho, maior será seu nível de comprometimento com a instituição.
\end{abstract}

\section{Palavras-chave}

Satisfação do professor. Compromisso social do professor. Condições do trabalho docente. Professores. Ensino básica. 


\title{
Job satisfaction and organizational commitment in teachers of the Peruvian Amazon
}

\begin{abstract}
This research sought to establish the relationship between job satisfaction and organizational commitment in teachers of regular basic education in the Peruvian Amazon. The research had a quantitative approach and its design was non-experimental of a correlational type. The sample consisted of 185 teachers to whom the Labor Satisfaction Scale and the Organizational Commitment Questionnaire were applied, instruments with the required validity and reliability. The findings indicate that teachers' level of job satisfaction and organizational commitment is regular and the existence of a direct and significant relationship between job satisfaction and organizational commitment was determined ( $r s=0.539 ; p=0.000<0.05$ ). It is concluded that the more satisfied teachers are in their work centers, the greater their level of commitment to the institution.
\end{abstract}

\section{Keywords}

Teacher's satisfaction. Teacher's social commitment. Teaching work conditions. Teachers. Elementary school.

\section{Introducción}

La satisfacción laboral es una variable que ha sido bastante estudiada en las últimas décadas en el campo de la psicología laboral (CERNAS; MERCADO; LEÓN, 2019) debido a sus implicancias en variables como la efectividad de la organización, el ausentismo, el desempeño y el compromiso organizacional (PUJOL; DABOS, 2018) y también porque constituye un factor que genera problemas cruciales dentro de las organizaciones (QUISPE; PAUCAR, 2020).

Según Herzberg (1954), la satisfacción laboral parte de las circunstancias extrínsecas a las motivaciones de las personas. Por ello, si una organización tiene la intención de crecer de manera estratégica y sobresalir entre la competencia, tiene que conocer a sus trabajadores y brindarles las condiciones básicas para que cumplan con sus responsabilidades: salarios acordes a la labor que realizan, ambientes seguros y dignos para que puedan desempeñarse adecuadamente y un trato justo (SÁNCHEZ; GARCÍA, 2017).

Muy a pesar de que la satisfacción laboral ha sido estudiada durante mucho tiempo, su concepto ha sido desarrollado de diferentes maneras de acuerdo a las concepciones de cada autor (CHIANG; OJEDA, 2013). Entre las principales conceptualizaciones, se destaca la de Locke (1976), quien lo definió como un estado 
emocional favorable o positivo que surge a partir de la percepción subjetiva de las experiencias laborales que tiene el trabajador. Del mismo modo, Bravo, Peiró y Rodríguez (1996) conceptualizaron la satisfacción laboral como un conjunto de actitudes que las personas desarrollan hacia su situación laboral, las cuales pueden referirse hacia la labor que desempeñan de manera general o hacia facetas específicas que realizan. Por otra parte, Robbins (2004) lo define como la actitud global de la persona hacia su trabajo.

Bajo esa perspectiva, se desprende que la satisfacción laboral es un factor que refleja el estado emocional de los trabajadores con su centro de labores. Por ello, implica una serie de emociones que ellos sienten sobre la labor que desempeñan (satisfacción intrínseca) y sobre los aspectos generales y del contexto de la institución (satisfacción extrínseca), las cuales determinan su bienestar, su compromiso con las labores que realizan y su productividad (CHIANG; GÓMEZ; HIDALGO, 2017).

En el caso de los profesionales de la educación, la existencia de insatisfacción laboral puede influir en las condiciones académicas, administrativas y personales. En el ámbito académico, puede afectar principalmente el desempeño que puedan tener en el aula, así como en su actualización y perfeccionamiento profesional. En el aspecto administrativo, podría reflejarse en las tardanzas, ausentismos, renuncias, etc. Por último, en el ámbito personal, podría verse reflejado en su autoestima, estrés y otros trastornos somáticos (DUCHE; GUTIÉRREZ; PAREDES, 2019). De lo expuesto, puede deducirse que "[...] la satisfacción o insatisfacción tendrán lugar cuando el trabajador se sienta complacido o no con su desempeño profesional, condicionado al mismo tiempo por las interacciones en el centro de trabajo y en el entorno laboral" (CANTÓN; TÉLLEZ, 2016, p. 217).

Con relación al compromiso organizacional, también es una variable bastante estudiada en el campo de la gestión de recursos humanos y la administración (ESTRADA; MAMANI, 2020), ya que es importante para el desarrollo personal del trabajador, para su desenvolvimiento profesional y el desarrollo organizacional (NEVES et al., 2018), así como por su relación con los principales factores del éxito de las organizaciones (BAEZ et al., 2019). Por ello, en la actualidad las organizaciones, a través de las oficinas de recursos humanos, procuran conocer cuán comprometidos están sus trabajadores para analizar su nivel de lealtad (CHIANG; GÓMEZ; WACKERLING, 2016; PEDRAZA, 2020).

Al igual que la satisfacción, el compromiso organizacional ha sido estudiado por diversos autores, por lo que existe una variedad de definiciones. Sin embargo, una 
definición bastante concreta la formularon Allen y Meyer (1996), quienes además fueron los autores que formularon un modelo teórico multidimensional sobre el mismo: lo definieron como el vínculo psicológico que tiene el trabajador con la organización, lo que permite crear lazos afectivos entre ambos actores, promoviendo además su permanencia.

En ese sentido y de acuerdo al modelo multidimensional desarrollado por Meyer y Allen (1991), el compromiso organizacional está asociado a la relación afectiva de los trabajadores con la organización (compromiso afectivo), la percepción de una obligación moral de permanecer en la organización por lealtad (compromiso de continuidad) y el costo-beneficio relacionado con su retiro de la misma (compromiso normativo). Es este modelo, ha promovido muchos estudios y ha sido ampliamente aceptado por los investigadores.

Posteriormente Aldana, Tafur y Leal (2018) asociaron al compromiso organizacional con el sentimiento que promueve en los trabajadores orgullo por la organización donde labora, a su vez evita que él piense en buscar otras oportunidades de trabajo. En ese sentido, se puede ver que, mediante el compromiso organizacional, se afianza la retención y atracción del personal debido a adecuadas condiciones laborales que le brindan, así como una positiva imagen corporativa.

De acuerdo a Estrada y Mamani (2020), el compromiso organizacional está relacionado de manera directa con el sentido de pertenencia, así como la responsabilidades que tienen los trabajadores con su organización, por ende, es menester contar con trabajadores comprometidos para que brinden una eficacia y eficiencia en la organización y así pueda incrementarse la productividad.

En el Perú, las condiciones laborales con las que los docentes se han afrontado han sido pésimas. De acuerdo a un informe técnico del Consejo Nacional de Educación (2014), los docentes tienen limitaciones en cuanto a las condiciones laborales. Entre las principales, se destacan que el $60,5 \%$ de docentes no cuentan con material educativo, lo cual limita su práctica pedagógica, dedican aproximadamente 12 horas adicionales a su jornada laboral estipulada en su contrato con la finalidad de preparar sus clases y los materiales, realizar reuniones con los padres de familia, etc.; y, en cuanto a su remuneración, el 66,3\% de ellos se encuentran insatisfechos con sus remuneraciones, por lo que optarían por buscar trabajos alternos para satisfacer las necesidades de sus familias (SILVA; MAIA FILHO; RABELO, 2019). 
Este problema se acrecienta en la zona rural, donde aproximadamente el $80 \%$ de los docentes consideran que los ambientes de trabajo se encuentran en mal estado. Esta situación, sumada a las relaciones interpersonales que se generan con el equipo directivo y a la constante carga burocrática traducida en los múltiples informes que presentan, estaría generando que no se sientan plenamente identificados con las instituciones donde trabajan ni desarrollen su sentido de pertenencia. A pesar que en los últimos años el Estado ha invertido en políticas de revalorización docente, todavía no han tenido los efectos esperados (AVELAR; SILVA; NASCIMENTO, 2019; LIMA; MENDES, 2019).

Al respecto, existen diversas investigaciones que se han realizado, tanto con docentes de educación básica regular como de educación superior, las cuales concluyeron que la satisfacción que perciban está relacionada con el compromiso y la identificación hacia su institución educativa (CHIARA, 2018; DUCHE; GUTIÉRREZ; PAREDES, 2019; INGA; SEGURA, 2019; JAIK; TENA; VILLANUEVA, 2010; MAMANI, 2016; MUHAMMAD; IMRAN, 2018; QUISPE; PAUCAR, 2020; RUIZ; SILVERA, 2019; VALDIVIA, 2018). Frente a ello, plantearon que era necesario que el Estado brinde las condiciones necesarias que dignifiquen la carrera docente, provea las adecuadas condiciones estructurales, mejore las políticas remunerativas y beneficios laborales, reduzca la carga administrativa para que se enfoquen estrictamente en la enseñanza de calidad y promueva su desarrollo personal y profesional.

En función a lo expuesto, la pregunta de investigación indica ¿Qué relación existe entre la satisfacción laboral y el compromiso organizacional en los docentes de educación básica regular de la Amazonía peruana? Para responder a dicha interrogante, se planteó el objetivo general, el cual busca establecer la relación entre la satisfacción laboral y el compromiso organizacional, mientras que los objetivos específicos buscan determinar la relación entre la satisfacción laboral y las dimensiones compromiso afectivo, de continuidad y normativo.

\section{Metodología}

\subsection{Diseño}

El enfoque de investigación fue cuantitativo, puesto que para probar la hipótesis se procedió a recoger datos, lo que hizo posible el análisis estadístico, así como la

Educ. Form., Fortaleza, v. 6, n. 1, e3854, jan./abr. 2021

DOI: https://doi.org/10.25053/redufor.v6i1.3854

https://revistas.uece.br/index.php/redufor/index ISSN: 2448-3583 
medición numérica de la información obtenida y sistematizada por los instrumentos aplicados (SÁNCHEZ; REYES; MEJÍA, 2018); el diseño fue no experimental, ya que ambas variables no fueron manipuladas deliberadamente, solo fueron observadas tal como se dieron en su entorno y luego se analizaron (HERNÁNDEZ; FERNÁNDEZ; BAPTISTA, 2014); y el tipo de investigación fue correlacional, ya que se buscó hallar la relación existente entre las dos variables (BISQUERRA, 2009).

\subsection{Población y muestra}

La población estuvo conformada por 357 docentes de educación básica regular pertenecientes a cinco instituciones educativas de la ciudad de Puerto Maldonado (Carlos Fermín Fitzcarrald, Dos de Mayo, Guillermo Billinghurst, Las Mercedes y Augusto Bouroncle Acuña) y la muestra fue constituida por 185 docentes, cantidad que fue hallada a través de un muestreo probabilístico estratificado. Del total de participantes, el $36,2 \%$ tenían entre 41 y 50 años, el $26 \%$ tenían entre 31 y 40 años, el 23,2 \% tenían más de 50 años y el 14,6 \% tenían entre 21 y 30 años. En cuanto al sexo, participaron más mujeres $(53,5 \%)$ que hombres (46,5\%). Respecto a la condición laboral, la mayoría de los docentes era nombrado $(68,1 \%)$ y el otro grupo contratado $(31,9 \%)$. Finalmente el $55,7 \%$ de los participantes eran docentes del nivel de educación primaria y el $44,3 \%$ eran docentes de educación secundaria. En la tabla 1 se detallan las características sociodemográficas de la muestra descritas previamente.

Tabla 1 - Características de la muestra

\begin{tabular}{llcc}
\hline & Características sociodemográficas & $\mathrm{n}=185$ & $\%$ \\
\hline \multirow{4}{*}{ Edad } & Entre 21 y 30 años & 27 & 14,6 \\
& Entre 31 y 40 años & 48 & 26,0 \\
& Entre 41 y 50 años & 67 & 36,2 \\
\multirow{2}{*}{ Sexo } & Más de 50 años & 43 & 23,2 \\
& Hombre & 86 & 46,5 \\
\multirow{2}{*}{ Condición laboral } & Mujer & 99 & 53,5 \\
\hline \multirow{2}{*}{ Nivel que enseña } & Nombrado & 126 & 68,1 \\
& Contratado & 59 & 31,9 \\
\hline
\end{tabular}

Fuente: Elaboración propia (2020). 


\subsection{Instrumentos}

Los instrumentos utilizados para la recolección de datos fueron la Escala de Satisfacción Laboral y el Cuestionario de Compromiso Organizacional.

La Escala de Satisfacción Laboral fue diseñada por Palma (1999). Está constituida por 36 ítems de tipo Likert (totalmente de acuerdo, de acuerdo, ni en acuerdo ni en desacuerdo, en desacuerdo y totalmente en desacuerdo) y evalúa siete dimensiones: condiciones físicas o materiales (ítems del 1 al 5), beneficios laborales 0 remunerativos (ítems del 6 al 9), políticas administrativas (ítems del 10 al 14), relaciones sociales (ítems del 15 al 18), desarrollo personal (ítems del 19 al 24), desempeño de tareas (ítems del 25 al 30) y relación con la autoridad (ítems del 31 al 36). La validez de contenido fue realizada mediante la técnica de juicio de expertos, para la cual se recurrió a cinco especialistas en administración educativa, obteniéndose un coeficiente $\mathrm{V}$ de Aiken de 0,947 , lo que indica que la escala presenta muy buena validez. En cuanto a la confiabilidad, fue determinada a través de una prueba piloto, obteniéndose un coeficiente Alfa de Cronbach de 0,951 , lo que significa que la escala es altamente confiable.

Con relación al Cuestionario de Compromiso Organizacional, fue elaborado por Meyer y Allen (1991) y adaptado a la realidad educativa peruana por Rivera (2010). Está conformado por 21 ítems de tipo Likert (totalmente de acuerdo, de acuerdo, ni en acuerdo ni en desacuerdo, en desacuerdo y totalmente en desacuerdo) y evalúa tres dimensiones: compromiso afectivo (ítems del 1 al 7), compromiso de continuidad (ítems del 8 al 15) y compromiso normativo (ítems del 16 al 21). El cuestionario presentó validez de contenido, ya que el coeficiente $V$ de Aiken obtenido a partir de la evaluación de los expertos fue de 0,919 y, en cuanto a la confiabilidad, alcanzó un coeficiente Alfa de Cronbach de 0,932 a partir de la prueba piloto, lo que significa que el cuestionario es altamente confiable.

\subsection{Procedimiento}

La recolección de datos fue realizada entre los meses de marzo y abril del año 2018. Antes de realizar la aplicación de los instrumentos, fue necesario solicitar la autorización de las instituciones educativas y el consentimiento de los docentes para que puedan ser partícipes de la investigación. Una vez obtenidas las autorizaciones, se procedió a aplicar ambos instrumentos por un lapso de 45 minutos.

Educ. Form., Fortaleza, v. 6, n. 1, e3854, jan./abr. 2021

DOI: https://doi.org/10.25053/redufor.v6i1.3854

https://revistas.uece.br/index.php/redufor/index ISSN: 2448-3583 


\subsection{Análisis estadístico}

Para realizar el análisis estadístico, se recurrió al Paquete Estadístico para las Ciencias Sociales (Statistical Package for the Social Sciences - SPSS), versión 22. Los resultados fueron categorizados y sistematizados mediante tablas de frecuencia y porcentaje. Para el análisis inferencial, se utilizó la prueba no paramétrica rho de Spearman debido a la naturaleza, nivel de medición y bondad de ajuste de las variables. Finalmente, para la significancia estadística, se consideró un valor menor o igual a 0,05.

\section{Resultados}

Los resultados que se presentan a continuación parten de la recolección de datos que fue efectuada a los docentes de educación básica regular de cinco instituciones educativas de la Amazonía peruana.

En la tabla 2 se puede ver que el $42,1 \%$ de los docentes presentan un nivel de satisfacción laboral regular, el 23,2 \% tienen un nivel bajo, el 22,2 \% presentan un nivel alto, el 7,6 \% evidencian un nivel muy alto y el $4,9 \%$ tienen un nivel muy bajo. Estos datos indican que casi la mitad de los docentes no se encuentran plenamente satisfechos con las condiciones laborales en las que se desenvuelven. Por otro lado, el $35,1 \%$ de los docentes presentan un nivel de compromiso organizacional regular, el $28,1 \%$ tienen un nivel alto, el 19,5\% evidencian un nivel bajo, el 10,8 \% presentan un nivel muy alto y el $6,5 \%$ tienen un nivel muy bajo. Lo expuesto es preocupante, porque revela que muchos de los docentes no se identifican con las instituciones donde laboran ni han desarrollado un vínculo afectivo hacia ellas.

Tabla 2 - Resultados generales de la satisfacción laboral y el compromiso organizacional

\begin{tabular}{lcccc}
\hline \multirow{2}{*}{ Niveles } & \multicolumn{2}{c}{ Satisfacción laboral } & \multicolumn{2}{c}{ Compromiso organizacional } \\
\cline { 2 - 5 } & $\mathrm{n}$ & $\%$ & $\mathrm{n}$ & $\%$ \\
\hline Muy bajo & 9 & 4,9 & 12 & 6,5 \\
Bajo & 43 & 23,2 & 36 & 19,5 \\
Regular & 78 & 42,1 & 65 & 35,1 \\
Alto & 41 & 22,2 & 52 & 28,1 \\
Muy alto & 14 & 7,6 & 20 & 10,8 \\
Total & 185 & 100,0 & 185 & 100,0 \\
\hline
\end{tabular}

Fuente: Elaboración propia (2020).

Educ. Form., Fortaleza, v. 6, n. 1, e3854, jan./abr. 2021

DOI: https://doi.org/10.25053/redufor.v6i1.3854

https://revistas.uece.br/index.php/redufor/index 
En la tabla 3 se puede ver que el p-valor de la prueba Kolmogorov-Smirnov para las variables satisfacción laboral y compromiso organizacional es inferior al nivel de significancia $(p=0,000<0,05)$, lo cual indica que los datos no presentan una distribución normal. En ese entender, fue necesario trabajar con una prueba estadística no paramétrica, siendo elegida en este caso la prueba rho de Spearman.

Tabla 3 - Prueba de normalidad Kolmogorov-Smirnov

\begin{tabular}{lccc}
\hline \multirow{2}{*}{ Variables } & \multicolumn{3}{c}{ Kolmogorov-Smirnov $^{\mathrm{a}}$} \\
\cline { 2 - 4 } & Estadístico & gl. & Sig. \\
\hline Satisfacción laboral &, 112 & 185 &, 000 \\
Compromiso organizacional &, 131 & 185 &, 000 \\
\hline
\end{tabular}

Fuente: Elaboración propia (2020).

De acuerdo a la tabla 4, el coeficiente de correlación rho de Spearman fue de 0,539 con un $p$-valor menor que el nivel de significancia $(p=0,000<0,05)$, lo cual nos indica que la satisfacción laboral está relacionada directa y significativamente con el compromiso organizacional, es decir, mientras los docentes presenten mayores niveles de satisfacción con las condiciones laborales, su nivel de compromiso y sentido de pertenencia hacia la institución educativa será mayor.

Tabla 4 - Correlación entre la satisfacción laboral y el compromiso organizacional

\begin{tabular}{cclc}
\hline & & Compromiso organizacional \\
\hline Rho de & Satisfacción & Coeficiente de correlación &, $539^{* *}$ \\
Spearman & laboral & Sig. (bilateral) &, 000 \\
& $N$ & 185 \\
\hline
\end{tabular}

${ }^{* *}$ La correlación es significativa en el nivel 0,01 (dos colas).

Fuente: Elaboración propia (2020).

En la tabla 5 podemos observar que existe relación directa y significativa entre la satisfacción laboral y las dimensiones compromiso afectivo ( $r s=0,601 ; p<0,05)$, compromiso de continuidad ( $r s=0,457 ; p<0,05)$ y compromiso normativo ( $r s=0,559$; $p<0,05)$.

Tabla 5 - Correlación entre la satisfacción laboral y el compromiso afectivo, de continuidad y normativo

\begin{tabular}{lcc}
\hline \multicolumn{1}{c}{ Dimensiones } & Rho de Spearman & p-valor \\
\hline Compromiso afectivo &, $601^{* *}$ &, 000 \\
Compromiso de continuidad &, $457^{* *}$ &, 000 \\
Compromiso normativo &, $559^{* *}$ &, 000 \\
\hline
\end{tabular}

${ }^{* *}$ La correlación es significativa en el nivel 0,01.

Fuente: Elaboración propia (2020).

Educ. Form., Fortaleza, v. 6, n. 1, e3854, jan./abr. 2021

DOI: https://doi.org/10.25053/redufor.v6i1.3854

https://revistas.uece.br/index.php/redufor/index 


\section{Discusión}

Un primer hallazgo encontrado indica que el $42,1 \%$ de los docentes presentan un nivel de satisfacción laboral regular, lo cual significa que ellos se caracterizan por tener una actitud poco positiva sobre su trabajo, la cual estaría condicionada principalmente por la precariedad de los ambientes de la institución educativa, la compensación económica que reciben, las relaciones interpersonales que se establecen en su centro de labores, la valoración del trabajo que realizan y la percepción del trato que reciben por parte de las autoridades educativas.

Estos resultados coinciden con lo reportado por Estrada, Mamani y Chura (2019), quienes investigaron el nivel de satisfacción de los docentes de educación básica del distrito de Tahuamanu, llegando a concluir que el 79,7\% de los docentes que participaron en la investigación presentaban un nivel de satisfacción laboral parcialmente alto, es decir, presentaban sentimientos poco favorables hacia sus instituciones educativas y tenían una limitada identificación con la misión, visión y objetivos institucionales.

Asimismo, guarda cierta relación con los hallazgos de Quispe y Paucar (2020), quienes investigaron cuán satisfechos se encontraban los docentes de una universidad peruana, concluyendo que $48,3 \%$ de ellos no se encontraban satisfechos debido a las pésimas condiciones en las que se desempeñaban traducidas en las bajas remuneraciones salariales, la poca promoción y nombramiento, las escasas oportunidades de crecimiento profesional y personal así como el poco reconocimiento que tenían por parte de las autoridades.

Por otro lado, coincide con los resultados obtenidos por Chaparro et al. (2017), quienes estudiaron la satisfacción laboral del personal docente y administrativo en instituciones de educación superior de México, identificando que la percepción que tenían los participantes sobre su nivel de satisfacción era intermedia, es decir, a pesar que se encontraban gustosos por laborar en la institución debido a su prestigio, existían sensaciones de insatisfacción por la remuneración que recibían, pues consideraban que no era coherente con la labor que desempeñaban.

Otro hallazgo indica que el $35,1 \%$ de los docentes presentan un nivel de compromiso organizacional regular, es decir, tienen poca identificación y sentido de pertenencia con la institución educativa donde laboran, por lo que no apuntan a aportar 
mediante sus acciones con los objetivos institucionales y, si encontraran otras opciones de trabajo, analizarían su permanencia en la institución.

Los resultados expuestos coinciden con los reportes de Maldonado et al. (2014), quienes investigaron sobre el compromiso organizacional en los docentes de una universidad pública de México. Concluyeron que el compromiso de los docentes se encontraba en un nivel medio, por lo que era necesario diseñar estrategias que busquen fomentar actitudes y comportamientos favorables en los docentes que contribuyan a consolidar su sentido de pertenencia.

Sin embargo, los hallazgos encontrados divergen de lo reportado por Estrada y Mamani (2020), quienes investigaron acerca del compromiso organizacional en docentes de instituciones educativas de educación básica en el Perú, llegando a la conclusión que los docentes se caracterizaban por presentar altos niveles de compromiso organizacional, es decir, se identificaban con la institución donde laboraban, involucrándose en las actividades institucionales, y, si hubiera otras opciones de trabajo, no las tomarían en cuenta, pues se encontraban complacidos de laborar en sus instituciones educativas.

Otro hallazgo relevante indica que la satisfacción laboral está relacionada directa y significativamente con el compromiso organizacional, es decir, mientras los docentes presenten mayores niveles de satisfacción, su nivel de compromiso y sentido de pertenencia hacia la institución educativa donde laboran también serán mayores. Este hallazgo coincide con los resultados obtenidos por investigaciones realizadas en docentes de educación básica regular (CHIARA, 2018; INGA; SEGURA, 2019; MAMANI, 2016; RUIZ; SILVERA, 2019) y de educación universitaria (DUCHE; GUTIÉRREZ; PAREDES, 2018; JAIK; TENA; VILLANUEVA, 2010; MUHAMMAD; IMRAN, 2018; QUISPE; PAUCAR, 2020; VALDIVIA, 2018), quienes encontraron que los profesionales de la educación que están a gusto con las condiciones laborales y salariales que les brindan las instituciones donde laboran suelen identificarse y trabajar de manera comprometida en búsqueda de la concreción de los objetivos institucionales.

En ese orden de ideas, la satisfacción laboral, tal como menciona Zubiri (2013), viene a ser una variable que nace a partir de la diferencia entre el trabajo real y las expectativas que tiene el trabajador, por ello es necesario que las instituciones generen las condiciones, brindando un ambiente físico y un entorno emocional en que se labore con tranquilidad. Del mismo modo, es importante que se reconozcan y valoren los logros 
que los trabajadores obtengan y se brinden las adecuadas compensaciones salariales para que los trabajadores puedan desempeñarse identificándose con la misión y visión de la institución y así se puedan concretizar sus objetivos.

Del mismo modo, estudios como el de Quispe y Paucar (2020) señalan que los trabajadores de una institución que se encuentran satisfechos presentan mejores niveles de compromiso, ya que evidencian mayores actitudes positivas hacia su trabajo, incluso por encima de las expectativas normales. A su vez, un trabajador comprometido también tendrá la disposición de permanecer trabajando en su institución, compartirá las metas que se logren y además dará más tiempo de lo establecido por la normatividad para apoyar a las labores curriculares y extracurriculares que la institución demande (PORTILLA, 2017).

Finalmente se demostró la existencia de una relación directa y significativa entre la satisfacción laboral y las dimensiones compromiso afectivo, de continuidad y normativo. Estos resultados indican que los adecuados niveles de satisfacción en los trabajadores están asociados a altos niveles de compromiso traducidos en la existencia de un lazo emocional que ellos manifiestan hacia su centro de trabajo, lo que fomenta, a su vez, su permanencia y continuidad en la institución y crea un sentimiento de gratitud por promover su crecimiento personal y profesional.

Este hallazgo coincide con lo reportado por Mamani (2016), quien determinó que la satisfacción laboral de los docentes de instituciones educativas adventistas se relaciona con las dimensiones del compromiso organizacional (afectivo, de continuidad y normativo).

\section{Consideraciones finales}

La satisfacción laboral es una variable que ha sido bastante estudiada en el ámbito organizacional, puesto que precisamente refleja cómo es su funcionamiento. Asimismo, su medición permite obtener los niveles de bienestar que presentan los trabajadores y está asociada de manera directa con el desempeño que tienen en su centro de trabajo. En ese sentido, la principal conclusión a la que se llegó fue que se pudo establecer que la satisfacción laboral se relaciona directa y significativamente con el compromiso organizacional, es decir, una institución que brinda adecuadas condiciones laborales y salariales promueve en sus trabajadores mayores niveles de 
identificación y sentido de pertenencia. Sin embargo, las instituciones que no generen las condiciones para que el trabajador esté satisfecho fomentarán en ellos indiferencia hacia las actividades que realizan y promoverá una constante rotación.

Por otro lado, se determinó que la satisfacción se relaciona también de manera directa con las dimensiones del compromiso organizacional (afectivo, de continuidad y normativo). Finalmente se determinó que el nivel predominante de satisfacción laboral y del compromiso de los docentes es regular. En ese sentido, se recomienda a las autoridades educativas a mejorar las condiciones laborales y salariales para que los docentes estén satisfechos, se desenvuelvan dignamente, desarrollen el sentido de pertenencia a sus instituciones y puedan mejorar así su desempeño y, por ende, la calidad educativa.

\section{Referencias}

ALDANA, E.; TAFUR, J.; LEAL, M. Compromiso organizacional como dinámica de las instituciones del Sector Salud en Barranquilla - Colombia. Revista Espacios, Caracas, v. 39, n. 11, p.13-23, 2018. Disponible en:

https://www.revistaespacios.com/a18v39n11/a18v39n11p13.pdf. Accedido el: 10 abr. 2020.

ALLEN, N.; MEYER, J. Affective, continuance, and normative commitment to the organization: an examination of construct validity. Journal of Vocational Behavior, v. 49, n.3, p. 252-276, 1996. DOI: 10.1006/jvbe.1996.0043. Disponible en: https://www.sciencedirect.com/science/article/abs/pii/S0001879196900433. Accedido el: 10 abr. 2020.

AVELAR, M.; SILVA, Y.; NASCIMENTO, M. La formación docente y su relación con la inserción profesional: políticas docentes en la red municipal de Río de Janeiro.

Educación \& Formación, v. 4, n. 2, p. 179-196, 2019. DOI: 10.25053/redufor.v4i11.1031. Disponible en: https://revistas.uece.br/index.php/redufor/article/view/1031/1148. Accedido el: 20 sept. 2020.

BAEZ, R. et al. Modelo conceptual del compromiso organizacional en empresas cubanas. Ingeniería Industrial, v. 40, n. 1, p. 14-23, 2019. Disponible en: http://scielo.sld.cu/pdf/rii/v40n1/1815-5936-rii-40-01-14.pdf. Accedido el: 20 sept. 2020.

BISQUERRA, R. Metodología de la investigación educativa. 2. ed. Madrid: La Muralla, 2009.

BRAVO, M.; PEIRÓ, J.; RODRÍGUEZ, I. Satisfacción laboral. In: PEIRÓ, J.; PRIETO, F. (ed.). Tratado de psicología del trabajo. Madrid: Síntesis, 1996. p. 37-90. 
CANTÓN, I.; TÉLLEZ, S. La satisfacción laboral y profesional de los profesores. Revista Lasallista de Investigación, v. 13, n. 1, p. 214-226, 2016. DOI: 10.22507/rli.v13n1a18. Disponible en: http://www.scielo.org.co/scielo.php?pid=s1794-

44492016000100018\&script=sci_abstract\&tIng=es. Accedido el: 10 nov. 2020.

CERNAS, D.; MERCADO, P.; LEÓN, F. Satisfacción laboral y compromiso organizacional: prueba de equivalencia de medición entre México y Estados Unidos. Contaduría y Administración, v. 63, n. 2, p. 1-23, 2019. DOI:

10.22201/fca.24488410e.2018.986. Disponible en:

http://www.scielo.org.mx/scielo.php?pid=s0186-

10422018000200006\&script=sci_abstract. Accedido el: 10 nov. 2020.

CHAPARRO, E. et al. Satisfacción laboral en instituciones de educación superior en la Zona Metropolitana del Valle de Toluca, México, como factor de competitividad. Revista de Desarrollo Económico, v. 4, n. 10, p. 29-42, 2017. Disponible en:

http://www.ecorfan.org/bolivia/researchjournals/desarrollo_economico/vol4num10/revista _de_desarrollo_econ\%c3\%b3mico_v4_n10_3.pdf. Accedido el: 10 nov. 2020.

CHIANG, M.; GÓMEZ, N.; HIDALGO, J. Job satisfaction of Chilean workers. A model of structural equations. Cuadernos de Administración, v. 33, n. 57, p. 48-60, 2017. DOI: 10.25100/cdea.v33i57.4538.

CHIANG, M.; GÓMEZ, N.; WACKERLING, L. Compromiso organizacional del funcionario municipal rural de la Provincia de Nuble, Chile. Ciencia y Trabajo, v. 18, n. 56, p. 134138, 2016. DOI: 10.4067/S0718-24492016000200010. Disponible en: https://scielo.conicyt.cl/scielo.php?script=sci_arttext\&pid=s0718-24492016000200010. Accedido el: 10 nov. 2020.

CHIANG, M.; OJEDA, J. Estudio de la relación entre satisfacción laboral y el desempeño de los trabajadores de las ferias libres. Contaduría y Administración, v. 58, n. 2, p. 39-60, 2013. DOI: 10.1016/S0186-1042(13)71209-9. Disponible en:

https://www.sciencedirect.com/science/article/pii/S0186104213712099. Accedido el: 10 nov. 2020.

CHIARA, A. Satisfacción laboral y compromiso organizacional de los docentes en la Institución educativa "Túpac Amaru", Villa María del Triunfo. 2018. Tesis (Maestría en Educación) - Posgrado en Educación, Universidad César Vallejo, Lima, 2018.

CONSEJO NACIONAL DE EDUCACIÓN. ¿Qué nos dicen los docentes sobre sus condiciones de trabajo en la escuela?. Lima: Consejo Nacional de Educación, 2014. Disponible en: http://repositorio.minedu.gob.pe/handle/minedu/5298. Accedido el: 20 sept. 2020.

DUCHE, A.; GUTIÉRREZ, O.; PAREDES, F. Satisfacción laboral y compromiso institucional en docentes universitarios peruanos. Revista Conrado, Cienfuegos, v. 15, n. 70, p. 15-24, 2019. Disponible en: https://conrado.ucf.edu.cu/index.php/conrado/article/view/1099. Accedido el: 20 sept. 2020. 
ESTRADA, E.; MAMANI, H. Compromiso organizacional y desempeño docente en las Instituciones de Educación Básica. Revista Innova Educación, v. 2, n. 1, p. 132-146, 2020. DOI: 10.35622/j.rie.2020.01.008. Disponible en:

https://www.revistainnovaeducacion.com/index.php/rie/article/view/70. Accedido el: 20 oct. 2020.

ESTRADA, E.; MAMANI, H.; CHURA, J. Clima organizacional y satisfacción laboral: Caso de docentes de instituciones educativas de educación básica. Conocimiento para el Desarrollo, Chimbote, v. 10, n. 2, p. 221-226, 2019. DOI: 10.17268/CpD.2019.02.08. Disponible en: https://revista.usanpedro.edu.pe/index.php/cpd/article/view/384. Accedido el: 20 oct. 2020.

HERNÁNDEZ, R.; FERNÁNDEZ, C.; BAPTISTA, P. Metodología de la investigación. 6. ed. México: Mc Graw-Hill, 2014.

HERZBERG, F. Work of the nature of man. Cleveland: The Word, 1954.

INGA, G.; SEGURA, E. Satisfacción laboral y compromiso organizacional en docentes de las instituciones educativas secundarias Mater Admirabilis y Nicolás la Torre del distrito de José Leonardo Ortiz. 2019. Tesis (Licenciatura en Psicología) - Pregrado en Psicología, Universidad Nacional Pedro Ruíz Gallo, Lambayeque, 2019.

JAIK, A.; TENA, J.; VILLANUEVA, R. Satisfacción laboral y compromiso institucional de los docentes de posgrado. Diálogos Educativos, v. 19, p. 119-130, 2010. Disponible en: http://www.repositoriodigital.ipn.mx/handle/123456789/3310. Accedido el: 20 sept. 2020.

LIMA, J.; MENDES, A. Desarrollo profesional docente: apuntes sobre política pública de formación de profesores. Educação \& Formação, Fortaleza, v. 4, n. 1, p. 180-194. DOI: 10.25053/redufor.v4i10.848. Disponible en:

https://revistas.uece.br/index.php/redufor/article/view/848/1089. Accedido el: 20 sept. 2020.

LOCKE, E. The nature and causes of job satisfaction. Chicago: Rand McNally, 1976.

SILVA, M.; MAIA FILHO, O.; RABELO, J. Las condiciones de trabajo del profesor sustituto en la universidad contemporánea: explorando la realidad de una universidad pública. Educação \& Formação, Fortaleza, v. 5, n. 1, p. 215-234, 2019. DOI: 10.25053/redufor.v5i13.1002. Disponible en: https://revistas.uece.br/index.php/redufor/article/view/1002. Accedido el: 20 sept. 2020.

MALDONADO, S. et al. Compromiso organizacional de los profesores de una universidad pública. Conciencia Tecnológica, Aguascalientes, v. 47, p. 12-18, 2014. Disponible en: https://dialnet.unirioja.es/servlet/articulo?codigo=4749342. Accedido el: 20 sept. 2020. 
MAMANI, A. Compromiso organizacional y satisfacción laboral en profesores de las instituciones educativas adventistas de la ciudad de Arequipa. 2016. Tesis (Licenciatura en Administración) - Pregrado en Administración, Universidad Peruana Unión, Puno, 2016.

MEYER, J.; ALLEN, N. A three component conceptualization of organizational commitment. Human Resource Management Review, v. 1, p. 61-89, 1991.

MUHAMMAD, A.; IMRAN, B. The effect of job satisfaction on teacher's organizational commitment, with special reference to private sector universities of Punjab, Pakistan. International Journal of Scientific and Research Publications, v. 8, n. 3, p. 114-125, 2018. DOI: 10.29322/ijsrp.8.3.2018.p7519. Disponible en:

https://www.researchgate.net/publication/323752303_the_effect_of_job_satisfaction_on_t eacher\%27s_organizational_commitment_with_special_reference_to_private_sector_uni versities_of_punjab_pakistan. Accedido el: 20 sept. 2020.

NEVES, T. et al. Compromiso organizacional, cualidades psicométricas e invarianza del cuestionario de Meyer y Allen para enfermeros portugueses. Revista Latino-Americana de Enfermagem, Ribeirão Preto, v. 26, p. 1-11, 2018. DOI: 10.1590/15188345.2407.3021. Disponible en: https://www.scielo.br/scielo.php?script=sci_arttext\&pid=S0104$11692018000100345 \&$ Ing=en\&nrm=iso\&tlng=es. Accedido el: 20 sept. 2020.

PALMA, S. Elaboración y validación de una Escala de Satisfacción Laboral SL-SPC para trabajadores de Lima metropolitana. Revista Teoría e Investigación en Psicología, Lima, v. 9, n. 1, p. 27-34, 1999.

PEDRAZA, N. Satisfacción laboral y compromiso organizacional del capital humano en el desempeño en instituciones de educación superior. Revista Iberoamericana para la Investigación y el Desarrollo Educativo, Guadalajara, v. 10, n. 20, 2020. DOI: 10.23913/ride.v10i20.595. Disponible en: https://www.ride.org.mx/index.php/ride/article/view/595. Accedido el: 20 sept. 2020.

PORTILLA, R. Satisfacción y compromiso organizacional de los trabajadores de un hospital de salud mental. 2017. Tesis (Licenciatura en Psicología) - Pregrado en Psicología, Universidad de Ciencias Aplicadas, Lima, 2017.

PUJOL, L.; DABOS, G. Satisfacción laboral: una revisión de la literatura acerca de sus principales determinantes. Estudios Gerenciales, Cali, v. 34, n. 146, p. 3-18, 2018. DOI: 10.18046/j.estger.2018.146.2809. Disponible en: http://www.scielo.org.co/pdf/eg/v34n146/0123-5923-eg-34-146-00003.pdf. Accedido el: 20 sept. 2020.

QUISPE, R.; PAUCAR, S. Satisfacción laboral y compromiso organizacional de docentes en una universidad pública de Perú. Apuntes Universitarios, Lima, v. 10, n. 2, p. 64-83, 2020. DOI: 10.17162/au.v10i2.442. Disponible en:

https://www.redalyc.org/jatsRepo/4676/467662252006/html/index.html. Accedido el: 20 sept. 2020. 
RIVERA, O. Compromiso organizacional de los docentes de una institución educativa privada de Lima Metropolitana y su correlación con variables demográficas. 2010. Tesis (Maestría en Educación) - Posgrado en Educación, Pontificia Universidad Católica del Perú, Lima, 2010.

ROBBINS, S. Comportamiento organizacional. 10. ed. México: Pearson Educación, 2004.

RUIZ, R.; SILVERA, B. Satisfacción laboral y compromiso organizacional en la Institución Educativa Particular Educadores y Promotores para el Desarrollo S.R.L., de la ciudad de Cajamarca. 2019. Tesis (Licenciatura en Administración) - Pregrado en Administración, Universidad Privada del Norte, Cajamarca, 2019.

SÁNCHEZ, H.; REYES, C.; MEJÍA, K. Manual de términos en investigación científica, tecnológica y humanística. Lima: Universidad Ricardo Palma, 2018.

SÁNCHEZ, M.; GARCÍA, M. Satisfacción Laboral en los Entornos de Trabajo. Una exploración cualitativa para su estudio. Scientia Et Technica, v. 22, n. 2, p. 161-166, 2017. Disponible en: https://www.redalyc.org/pdf/849/84953103007.pdf. Accedido el: 20 sept. 2020.

VALDIVIA, L. La satisfacción laboral y el compromiso organizacional de los docentes de la Facultad de Ecoturismo de la Universidad Nacional Amazónica de Madre de Dios. 2018. Tesis (Maestría en Educación) - Posgrado en Educación, Universidad César Vallejo, Madre de Dios, 2018.

ZUBIRI, F. Satisfacción y motivación profesional. Anales del Sistema Sanitario de Navarra, Pamplona, v. 36, n. 2, p. 193-196, 2013. DOI: 10.4321/S113766272013000200002. Disponible en: http://scielo.isciii.es/scielo.php?script=sci_arttext\&pid=S1137-66272013000200002. Accedido el: 20 sept. 2020.

\footnotetext{
Edwin Gustavo Estrada Araoz, Universidad Nacional Amazónica de Madre de Dios, Institución Educativa Almirante Miguel Grau Seminario

i (Dhttps://orcid.org/0000-0003-4159-934X

Doctor en Educación, maestro en Administración de la Educación y Psicología Educativa, egresado de la Maestría en Políticas y Gestión de la Educación. Docente de pregrado en la Universidad Nacional Amazónica de Madre de Dios y en la Institución Educativa Almirante Miguel Grau Seminario.

Contribución de autoría: Concepción del texto.

E-mail: edwin5721@outlook.com
} 
Néstor Antonio Gallegos Ramos, Universidad Nacional Amazónica de Madre de Dios, Ingeniería de Sistemas

ii Dhttps://orcid.org/0000-0003-1436-9207

Doctor en Economía y Medio Ambiente, magíster en Ciencias de la Educación, ingeniero estadístico, especialista en procesamiento y análisis de datos. Docente nombrado en la Universidad Nacional Amazónica de Madre de Dios en la carrera de Ingeniería de Sistemas.

Contribución de autoría: Concepción del texto.

E-mail:ngallegor@gmail.com

Editora responsable: Lia Machado Fiuza Fialho

Pareceristas ad hoc: Hugo Heredia Ponce y Antonio Gutiérrez Rivero

\section{Cómo citar este artículo (ABNT):}

ESTRADA ARAOZ, Edwin Gustavo; GALLEGOS RAMOS, Néstor Antonio. Satisfacción laboral y compromiso organizacional en docentes de la Amazonía peruana. Educ. Form., Fortaleza, v. 6, n. 1, e3854, 2020. Disponible en:

https://revistas.uece.br/index.php/redufor/article/view/3854

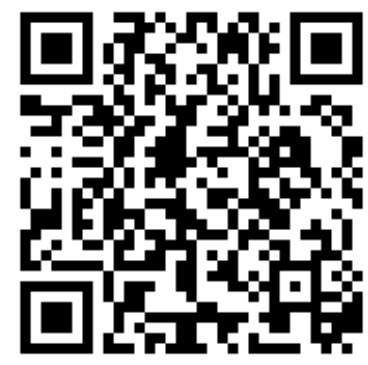

Recibido el 23 de agosto de 2020.

Aceptado el 14 de septiembre de 2020.

Publicado el 10 de noviembre de 2020. 